\title{
N-Myc Downstream-Regulated Gene 2 Induces Angiogenesis
}

\author{
Mina Song and Sayeon Cho \\ College of Phamacy, Chung-Ang Lniversin, Seoul 156-756, Korea. ${ }^{*}$ E-mail: sychöacau ac.kr \\ Received June 20, 2007
}

Key Words : N-Myc downstream-regulated gene 2 (NDRG2), Angiogenesis, Hypoxia-inducible factor-1 (HIF-1)

Angiogenesis is the process of generating new capillary blood vessels from pre-existing vessels and a fundamental process in the normal development, reproduction. and wound healing. Since neovascularization in physiological processes is tightly regulated by a balance of angiogenic and anti-angiogenic factors. disrupted balance effect play's a leading role in the progress of diseases such as tumor growth. rheumatoid arthritis. and various blood vesselrelated pathology. $!^{1-3}$ Tumor growth relies on angiogenesis to receive an adequate supply of nutrients and oxygen. In addition. the newly formed blood vessels provide a way for tumor cells to enter the circulation and to metastasize to distant organs. Therefore. treatment of cancer with antiangiogenic agents will control cancer cell growth and metastasis. Among a variety of angiogenic factors. vascular endothelial growth factor (VEGF) and basic fibroblast growth factor (bFGF) have a prominent activity in tumor metastasis and mortality. ${ }^{4}$ Specially. VEGF expression is induced in various cancers by several stimuli and its transcriptional activation is mediated by hỵpoxia-inducible factor-1 (HIF1). 56

Since angiogenesis requires a multi-step mechanism. the process is mediated by a wide range of angiogenic regulators. In an effort to search for endogenous angiogenic factors. we found that $\mathrm{N}-\mathrm{myc}$ downstream-regulated gene 2 (NDRG2) is involved in angiogenesis. Human NDRG2 belongs to a family of NDRG The human NDRG family includes four identified members. NDRG1. NDRG2. NDRG3. and NDRG4, which are highly homologous except for in the $\mathrm{C}$ - and N-terminal regions. ${ }^{7}$ NDRG2 initially identified as a candidate tumor suppressor gene since its expression is reduced in several cancer cells. ${ }^{8.5}$ It has also been suggested that NDRG2 may have roles in cell differentiation. ${ }^{10}$ Alzheimer's disease. ${ }^{11}$ and neurite overgrow th. "2 However. NDRG2 activity related to angiogenesis was not yet reported.

Since endothelial cell migration through extracellular matrix is one of essential steps for neovascularization. we tested the effect of conditioned medium derived from NDRG2-transfected cells (NDRG2-CM) on endothelial cell migration. To establish cell line expressing NDRG2, human embryonic kidney (HEK) 293 cells were transfected with NDRG2 expression plasmid and transfected cells stably expressing NDRG2 were selected as described in experimental section. After expression of NDRG2 was confinned by Western blotting analysis. conditioned medium (CM) from the selected cells was obtained. In chemotaxis chamber. human umbilical vein endothelial cells (HUVECs) were treated with $\mathrm{CM}$ from control transfected cells and stably transfected cells for $2 \mathrm{~h}$ and HUVECs that had been migrated through membrane pores were counted under microscope. NDRG2-CM showed significantly induced migratory effect. compared to control-CM (Fig. 1A and B). Therefore. these data indicate that NDRG2-CM has the biological activity that can induce endothelial cell migration.

We next examined the ability of NDRG2-CM to promote the mesh-like structures of HUVECs on matrigel. Matrigel is generally used for studying HUVEC attachment. migration and differentiation. The extent of mesh-like structure was more enhanced in HUVECs influenced in NDRG2-CM than that of control CM (Fig. 2A and B). This result indicates that NDRG2-CM has the ability of inducing tube fomation of endothelial cells.

We then measured the protein level of VEGF in NDRG2-

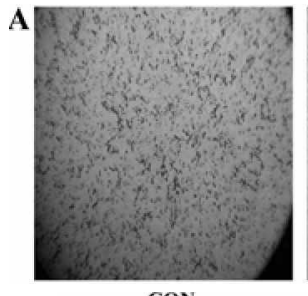

CON

H

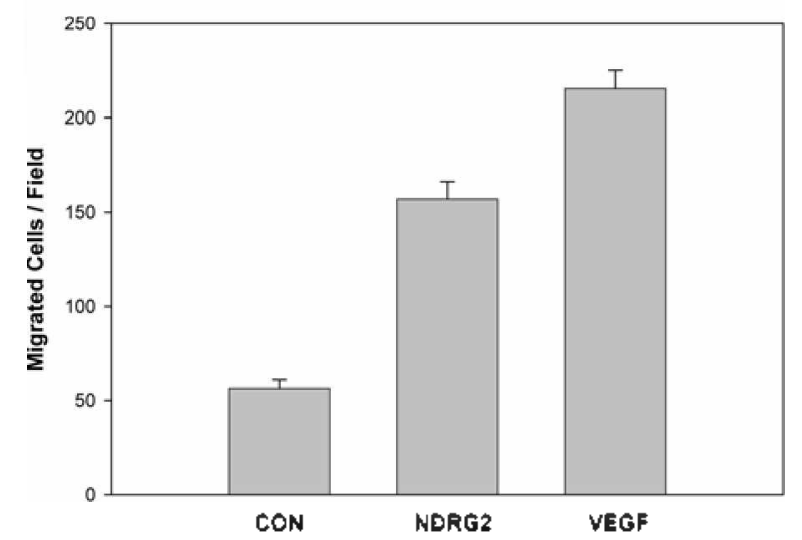

Figure 1. NDRG2-CM stimulates endothelial cell migration. (A) HUVEC's migration assay was carried out in 48 -well microchemotasis chambers. HUVECs were incubated with control-CM or NDRG2-CM for $2 \mathrm{~h}$. Representative images of cell migration are shown. (B) After fixation and staining, the number of migrated cells was determined by counting two regions of each well under a microscope. VEGF (20 $\mathrm{ng} / \mathrm{mL}$ ) was used as a positive control. 


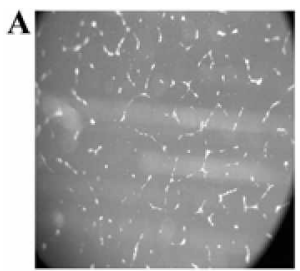

CON

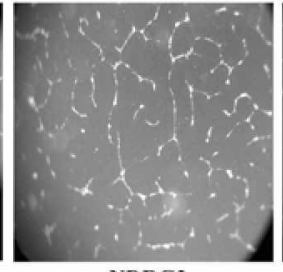

NDRG2

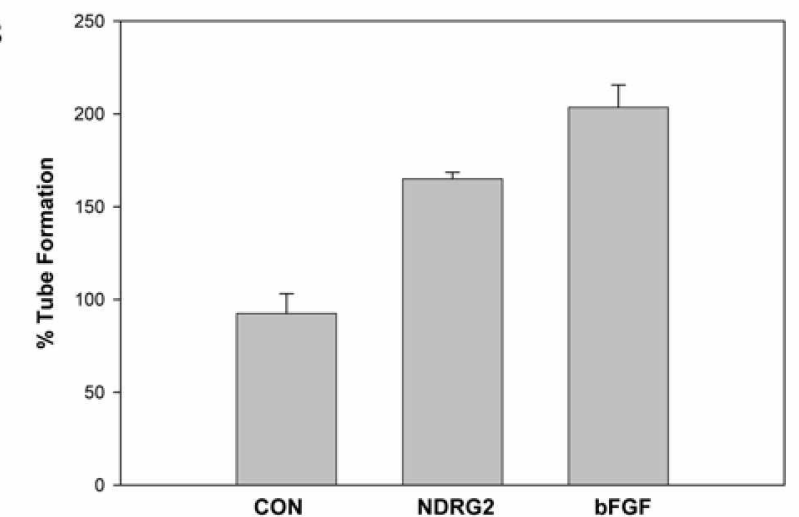

Figure 2. NDRG2-CM promotes tube formation of endothelial cells. (A) HUVECs were collected and seeded on Matrigel-coated plates at a density of $2.4 \times 10^{4}$ cells/well and then incubated with control-CM or NDRG2-CM. As a positive control, bFGF (10 ng/ $\mathrm{mLL}$ ) was used. After $18 \mathrm{~h}$, fields from each sample were photographed. Representative images of tube fonmation activity are shown. (B) Total tube areas were analyzed by the Scion Image program. The control tube areas were defined as 100\% tube fonmation, and the percent increase in tube fornation as compared with control was calculated for each sample.

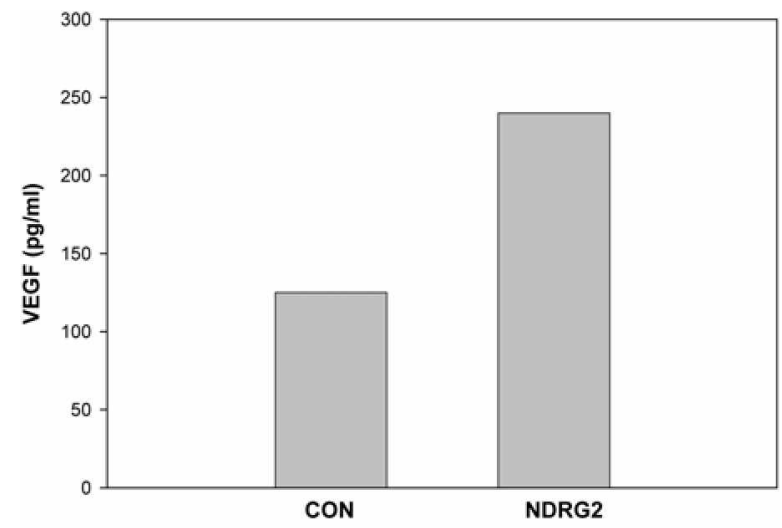

Figure 3. NDRG2 increases the level of VEGF in CM. Control or NDRG2-expressing stable cells were incubated in serunn-free M199 for $20 \mathrm{~h}$, and aliquots of the CM were collected. The level of VEGF in CM was measured by ELISA as described under experimental section.

$\mathrm{CM}$ to investigate whether VEGF production is involved in NDRG2-stimulated HUVEC tube formation since it is a major component of angiogenesis. Using ELISA assay kit. we found that NDRG2-overexpressing cells released significantly more VEGF than control cells (Fig. 3). This result suggests that the increased secretion of VEGF may be associated with NDRG2-induced angiogenic activity involved in migration and tube formation.

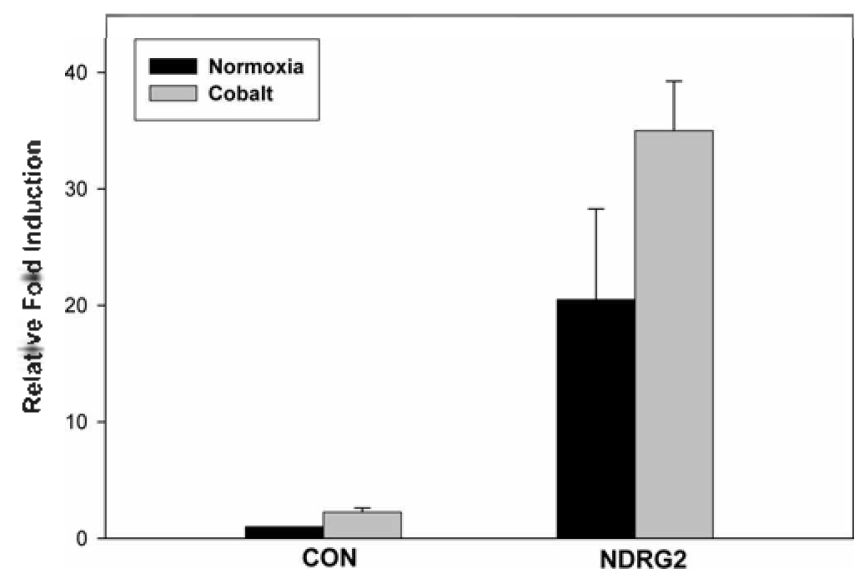

Figure 4. NDRG2 activates the transactivation function of HLF-1. HEK 293 cells that constitutively express NDRG2 and control cells that contain empty plasmid were transfected by lipofectamine with $1 \mathrm{tg}$ each of the reporter plasmid HRE-Luc and $0.5 \mathrm{tg}$ of pCMV/ $\beta$-gal. Cells were treated with $125 \mu \mathrm{M} \mathrm{CoCl}_{2}$ for $24 \mathrm{~h}$. Luciferase activities were normalized to $\beta$-galactosidase activities.

Since NDRG2-CM contains the increased level of VEGF, we examined whether NDRG2 regulates the transcriptional activity of HIF-l that is stabilized at hypoxia and plays a key role in VEGF transcription. NDRG2-overexpressing cells were transfected with pHRE-Luc reporter plasmid and either grown under nonmoxic condition or treated with a hypoxiamimicking reagent ( $\left.125 \mu \mathrm{M} \mathrm{CoCl}_{2}\right)$. As shown in Figure 4, NDRG2-overexpressing cells resulted in an increase of the luciferase activity under both nonmoxic and hypoxia-mimicking conditions. compared to control cells grown at normoxia or hypoxia. Interestingly, the relative ratio of luciferase activities from NDRG2-overexpressing cells incubated under hypoxia-mimicking and normoxic conditions is significantly lower than that of control cells. suggesting that NDRG2 activates HIF-l activity primarily at nomoxia. Taken together, these results suggest that NDRG2 activates the HIF-l $\alpha$ transcriptional activity and that its activatory function is oxygen-independent

In the present study, we found that NDRG2 induced angiogenesis although it is a candidate tumor suppressor gene due to its low expression in various cancer tissues. Interestingly: NDRG1 is highly expressed in many types of human tumors including colon. breast. prostate. kidney. liver and brain cancers compared with normal tissue. ${ }^{i 3}$ Moreover, NDRGI overexpression in some cancers shows a marked reduction in tumor-induced angiogenesis. ${ }^{14}$ suggesting that overexpression of proteins in cancer cells is not always favorable for cancer growth. The distinct expression patterns of NDRG1 and NDRG2 suggest that they have different functions in angiogenesis of cancer tissues. These findings support the possibility that NDRG2 has angiogenic activity. We confirmed that this ability of NDRG2 is involved in the increased VEGF levels and HIF-1 transactivation.

In conclusion, we demonstrated that NDRG2 mediates angiogenesis and VEGF may be the target of NDRG2-medi- 
ated process. NDRG2 could be a novel target for developing anti-cancer agent in tumor growth and angiogenesis.

\section{Experimental Section}

Cell culture. Human embryonic kidney (HEK) 293 and human unibilical vein endothelial cells (HUVECs) were routinely maintained as previously described. ${ }^{15.10}$

Plasmid construction. The N-terminal hemagglutinin (HA)-tagged NDRG2 gene for expression in mammalian cells was constructed by polymerase chain reaction. followed by cloning into the pcDNA3.1/HA Zeo plasmid. The luciferase reporter plasnid (HRE-Luc) containing the promoter of HIF-I $\alpha$ was purchased from Stratagene.

Stable transfection and Western blotting. HEK293 cells were transfected by lipofectamine (Invitrogen) with $1 \mu \mathrm{g}$ each of pcDNA3.1 Zeo-NDRG2 or pcDNA3.1 Zeo-empty vector. After $48 \mathrm{~h}$. transfected cells were selected for two weeks in DMEM containing 10\% FBS and zeocin $(200 \mu \mathrm{g} /$ $\mathrm{mL}$ ). After 15 days of selection. resistant cells arising in dishes were propagated in zeocin of low level $(150 \mu \mathrm{g} / \mathrm{mL})$. The expression of stably transfected genes was confirmed by Western blotting as previously described. ${ }^{10}$

NDRG2-CM preparation. Confluent stable cells were washed and grown in serum free M199 medium. After $20 \mathrm{~h}$ of incubation. CM was collected and centrifuged at $500 \times \mathrm{g}$ for $10 \mathrm{~min}$ and then at $800 \times \mathrm{g}$ for $20 \mathrm{~min}$ to remove debris. The resultant $\mathrm{CM}$ was immediately used for migration and tube formation assays or stored $-70^{\circ} \mathrm{C}$ until use

HUVEC migration and tube formation assays. Endothelial cell migration and tube formation assays were carried out as previously described. ${ }^{17}$

Determination of VEGF level. To determine VEGF protein expression level. we obtained $\mathrm{CM}$ from control or stable cells. The amount of VEGF protein found in the $\mathrm{CM}$ was determined as previously described. ${ }^{15}$

Luciferase assay. Stable cells were grown to $50-70 \%$ confluence in $60 \mathrm{~mm}$ dish. and were transfected by lipo- fectamine with $\mathrm{l} \mu \mathrm{g}$ pHRE-Luc and pCMV/ $\beta$-gal. and assayed as previously described. ${ }^{18}$

Acknowledgements. This Research was supported by the Chung-Ang University Research Grants in 2005.

\section{References}

1. Boehm-Viswanathan. T. Cum Opin. Oncol. 2000.12 .89$.

2. Carmeliet. P. Nat. Med. 2003. 9.653.

3. Carmeliet. P.: Jain. R. K. Nature 2000. 407. 249.

4. Yoshiji. H.: Kunvama, S. Yoshii, J.; Ikenaka. Y.: Noguchi, R: Hicklin, D. J; Huter. J.: Nakatani, T;; Tsujinoue. H.: Yanase. K: Imazu. H.: Fukui. H. Hepatolog, 2002, $35,834$.

5. Fukida. R.: Kelly. B.: Semenza. G. L. Cancer Res 2003. 63. 2330.

6. Ravi. R.: Mookerjee. B.: Bhujwalla. Z. M.: Sutter. C. H: Artemov. D.: Zeng. Q.: Dillehay. L. E.: Madan. A.: Semenza. G. L.; Bedi, A. Genes Den: 2000, 14, 34

7. Zhou. R. H.: Kokame, K.: Tsukamoto. Y: Yutani, C.: Kato. H. Miyata. T. Genomics 2001. 73.86.

8. Liu. N.: Wang. L.: Liu. X.: Yang. Q.: Zhang. T.: Zhang. W.: Wu. Y.: Shen1. L.: Zhang. Y.: Yan1g. A.: Han1. H.: Zhang. T.: Yao. L. Biochent. Biophs. Res Contmum. 2007.358. 164

9. Deng. Y: Yao. L.: Chau, L.; Ng. S. S.: Peng. Y.: Liu. X: Au, W. S.: Wang, J.: Li, F.; Ji. S.; Han. H.; Nie, X.: Li, Q.: Kung, H. F; Leung. S. Y; Lin, M. C. Int. J. Cancer 2003, 106, 342.

10. Choi. S. C.: Kim1. K. D.: Kim1. T. T.: Kim1. T. W.: Yoon. D. Y.: Choe. Y. K.: Chang. Y. S.: Paik. S. G.: Lim. T. S. FEBS Lett 2003. 553.413

11. Mitchelmore. C.: Buchmann-Moller. S.: Rask. L.: West. M. J.: Troncoso. J. C.: Jensen. N. A. Newrobiol. Dis. 2004. 16, 48

12. Takahashi, K.; Yamada. M.; Ohata, H.: Honda, K.: Yamada. M. Yeurosci. Lett. 2005, 388. 157

13. Cangul. H.: Salnikow: K.: Yee. H:: Zagzag. D.: Commes. T.: Costa. M. Cell Biol. Toricol 2002. 18.87.

14. Maruyama. Y: Ono. M.: Kawahara. A.: Yokoyama. T.: Basaki. Y.: Kage. M.: Aovagi, S.; Kinoshita. H.: Kuwano, M. Cancer Res. 2006. 66,6233

15. Lee. J. A.: Park. J. E.: Hwang, S.; Lee. D. H.; Park, S. G; Park, B C.: Cho. S. Bull. Korem Chem. Soc. 2006. 27. 1456

16. Cho. S. Bull. Korean Chem. Soc. 2005. 26. 1823.

17. Kim. C. W.: Lee. H. M.: Lee. I. H.: Kang. C.: Kleinnan. H. K. Gho. Y.S. Cancer Res. 2002. 62,6312.

18. Park, J. E.; Lee, D. H.; Lee, J. A.; Park, S. G.: Kim, N. S.: Park, B C. Cho, S. Biochem. Biophys. Res. Commun 2005. 337, 1283. 